\title{
L'APPLICATION DU CALCUL DES PROBABILITÉS AUX PROBLĖMES D'EXPLOITATION DES RÉSERVOIRS
}

\section{LA THÉORIE STOCHASTIQUE DES RÉSERVOIRS Application au problème des réservoirs destinés à I'atténuation des crues}

\author{
PAR \\ J. BERNIER *
}

Le rôle de plus en plus important joué par les barrages réservoirs dans le domaine de l'Economie de l'Eau rend nécessaire une étude précise de leur gestion et de leur capacité à atteindre les buts pour lesquels on les a projetés. La difficulté de cette étude tient essentiellement à la nature aléatoire des apports hydrauliques, de telle sorte que l'efficience de ces réservoirs ne peut être chiffrée qu'en termes de probabilité.

C'est ainsi qu'un réservoir destiné à la seule atténuation des crues peut ètre caractérisé par :

$1^{\circ}$ la probabilité $\mathrm{P}$ de dépassement du débit de sortie maximal au-dessus duquel des dommages importants peuvent survenir à l'aval;

$2^{\circ}$ la fréquence des périodes aux cours desquelles le réservoir est vide.

Si le réservoir est utilisé conjointement, soit à une accumulation d'eau en vue du soutien des étiages de la saison sèche (irrigation), soit à la production d'énergie hydroélectrique, il importe de mesurer notamment l'incidence de cette utilisation sur la probabilité P.

P. A. P. Moran dans sa "Theory of storage» (Methuen's Monographs, 1959) a bâti un cadre mathématique adéquat à la résolution de ces problèmes. L'application aux problèmes des crues en est cependant difficile car Moran suppose l'indépendance en probabilité des apports au cours de périodes successives, ce qui impose la prise en compte de périodes élémentaires d'assez longue durée, telles qu'on est conduit à les envisager dans l'étude des réservoirs interannuels, par exemple. Dans un article récent (A note on the solution of dam equations - Journal of the Royal Statistical Society, series B, vol. 26, $\mathbf{n}^{\circ} 2,1964$ ) Lloyd et Odoom établissent un algorithme permettant le calcul dans le cas d'apports successifs liés en chaîne pour le cas des variables discrètes.

Dans le présent rapport, après rappel des éléments de base de la théorie classique de Moran, nous voudrions donner quelques indications sur les modes de traitement (notamment la méthode de simulation) de modèles plus réalistes quant aux applications éventuelles. La principale qualité de la méthode de simulation est une très grande souplesse qui permet de l'adapter aux caractéristiques d'une grande variété de problèmes pratiques.

Dans certains cas, des méthodes plus analytiques sont cependant plus précises et plus économiques en temps de calcul sur machine électronique. A cet égard, nous voudrions donner des résultats qui semblent nouveaux concernant le cas des apports vérifiant un schéma particulier de liaison en probabilité qui semble suffisamment proche de la réalité pour la majorité des applications courantes. La fonction de répartition de la variable aléatoire : contenu du réservoir, s'exprime comme solution d'une certaine équation intégrale que, dans la pratique, il est commode de remplacer par un système d'équations linéaires dont la résolution est possible au moyen d'une calculatrice électronique.

\footnotetext{
* Ingénieur au Centre de Recherches et d'Essais de Chatou (E.D.F.).
} 


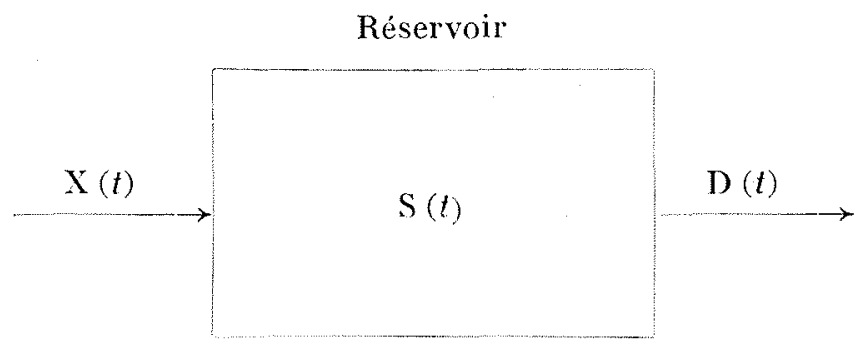

Le contenu d'un réservoir à l'instant $t$ est un processus aléatoire dont les valeurs positives ou nulles sont bornées supérieurement par la capacité du réservoir $\mathrm{K}$; ce processus est par ailleurs conditionné par le processus aléatoire $\mathrm{X}(t)$ du débit entrant et par le processus aléatoire $\mathrm{D}(t)$ du débit sortant dont la loi de probabilité est fonction de la règle de gestion fixée. Pour la simplicité, nous nous restreindrons dans la suite à l'étude des processus pour des suites d'instants discrets équidistants, repérés de façon conventionnelle par des entiers :

$$
\ldots, \mathrm{t}-1, t, t+1, \ldots
$$

De façon également conventionnelle, on peut admettre, d'une part, que la règle de gestion admise entraîne la vidange globale à la fin de la période $t$ d'un certain volume $D_{t}$ et, d'autre part, que le contenu du réservoir $Z_{i}$ est considéré au début de la période $t$ après la vidange de $\mathrm{D}_{t-1}$, et avant l'occurrence de l'apport $\mathrm{X}_{t}$ de la période $t$.

Le modèle simplifié proposé par Moran suppose que le contenu du réservoir est complètement vidangé dans la mesure où le volume sortant est inférieur à un certain seuil M. Dans le cas contraire, le volume sortant est fixé au niveau $M$ si la capacité maximale $K$ n'est pas atteinte; si elle l'est, il y a déversement entrainant un volume sortant supérieur au seuil $M$.

Formellement, on a les relations de récurrence suivantes :

$$
\begin{array}{lll}
Z_{t+1}=0 & \text { si } & X_{t}+Z_{t}<M \\
Z_{t+1}=Z_{t}+X_{t}-M & \text { si } & M \leqslant X_{t}+Z_{t}<K \\
Z_{t+1}=\mathrm{K}-\mathrm{M} & \text { si } & X_{t}+Z_{t} \geqslant K
\end{array}
$$

La prise en compte de plusieurs objectifs amène à des règles de gestion plus élaborées. Cependant, dans le cas du remplissage d'un réservoir destiné à l'irrigation et de l'exploitation d'un réservoir hydroélectrique, la règle de gestion peut être schématisée par la considération d'une « courbe d'alerte » qui, pour chaque période $t$, définit un seuil $k$ représentant le niveau de remplissage que l'on doit viser, en stockant si le contenu du réservoir lui est inférieur au début de la période, ou en vidangeant dans les limites admises (O, M) dans le cas contraire.

De facon plus formelle on peut écrire :

$$
\begin{array}{lll}
Z_{t+1}=Z_{t}+X_{t} & \text { si } \quad Z_{t}+X_{t}<k_{t} \\
Z_{t+1}=k_{t} & \text { si } \quad k_{t} \leqslant Z_{t}+X_{t}<k_{t}+\mathbf{M} \\
Z_{t+1}=Z_{t}+X_{t}-M & \text { si } \quad k_{t}+M \leqslant Z_{t}+X_{t}<\mathbf{K} \\
Z_{t+1}=\mathbf{K}-\mathrm{M} & \text { si } \quad Z_{t}+\mathrm{X}_{t} \geqslant \mathbf{K}
\end{array}
$$

Les formules (1) et (2), ainsi que toutes celles que l'on peut construire aisément sur la base de règles de gestion plus complexes, permettent la détermination de la loi de probabilité des états du processus $Z_{t}$ à l'instant $t$ quel que soit cet instant, à partir de la donnée de la loi de probabilité du processus $\mathrm{X}_{t}$. Avant d'en venir aux méthodes de résolution, nous voudrions rappeler quelques notions générales sur les variables aléatoires et les processus stochastiques.

\section{Rappel de quelques notions générales sur les variables aléatoires et les processus stochastiques}

La variable aléatoire $Z_{t}$ est du type mixte : continue par intervalles où elle possède une densité de probabilité, elle prend des probabilités finies pour un ensemble dénombrable de valeurs de la variable.

Soit $H(z)$ la fonction de répartition d'une variable aléatoire mixte $Z$ :

$$
H(z)=\operatorname{Prob}[Z \leqslant z]
$$




\section{J. BERNIER}

Cette fonction positive, bornée par 1 , a donc un ensemble dénombrable de discontinuités. Les grandeurs intervenant dans la suite seront de la forme :

$$
\mathrm{I}=\int_{a}^{b} g(z) d \mathrm{H}(z)
$$

Cette intégrale s'interprète comme la limite de la somme :

$$
\sum_{a}^{b} g\left(z_{i}\right)\left[\mathrm{H}\left(z_{i+1}\right)-\mathrm{H}\left(z_{i}\right)\right]
$$

où l'intervalle $(a, b)$ est scindé en intervalles partiels $\left(z_{i}, z_{i+1}\right)$ dont la plus grande longueur tend vers 0 . Si la limite existe, elle définit une intégrale de Stieltjes.

La quantité $\Delta \mathrm{H}=\mathrm{H}\left(z_{i+1}\right)-\mathrm{H}\left(z_{i}\right)$ est la mesure de probabilité de l'intervalle $\left(z_{i}, z_{i+1}\right)$.

Si $z_{i+1}$ est voisin de $z_{i}$, soit $z_{i+1}=z_{i}+d z_{i}$, alors on peut écrire sur tout intervalle de continuité :

$$
\Delta \mathrm{H} \simeq d \mathrm{H}=h\left(z_{i}\right) d z_{i}
$$

où $h\left(z_{i}\right)$ est la densité de probabilité de $Z$ sur tout intervalle de continuité. Par contre, en tout point de discontinuité :

$$
d \mathbf{H}=\operatorname{Lim}_{z_{i+1} \rightarrow z_{i}} \Delta \mathbf{H}=p_{i}
$$

où $p_{i}$ est la probabilité finie affectée à la valeur $z_{i}$.

La généralisation au cas de 2 variables $Z_{1}$ et $Z_{2}$ ou plus est aisée et on peut notamment définir la mesure de probabilité du rectangle :

$$
z_{1}^{i} \cdot z_{1}^{i+1}, z_{2}^{i} \cdot z_{2}^{i+1}
$$

à partir de la fonction de répartition du couple :

$$
\begin{gathered}
\mathrm{H}\left(z_{1}, z_{2}\right)=\operatorname{Prob}\left[Z_{1} \leqslant z_{1}, Z_{2} \leqslant z_{2}\right] \\
\Delta_{2} \mathrm{H}=\mathrm{H}\left(z_{1}{ }^{i+1}, z_{2}{ }^{i+1}\right)-\mathrm{H}\left(z_{1}^{i+1}, z_{2}{ }^{i}\right)-\mathbf{H}\left(z_{1}^{i}, z_{2}^{i+1}\right)+\mathrm{H}\left(z_{1}^{i}, z_{2}^{i}\right)
\end{gathered}
$$

La limite $d_{2} \mathrm{H}$ se définit aisément en généralisant les formules (4) et (5) :

- sur tout domaine de continuité de $\mathrm{H}$ :

$$
d_{22} \mathrm{H}=\frac{\partial^{2} h\left(z_{1}, z_{2}\right)}{\partial z_{1} \partial z_{2}} d z_{1} d z_{2}
$$

- pour tout point de discontinuité $\left(z_{1}, z_{2}\right): d_{2} \mathrm{H}=\mathrm{P}_{12}$ probabilité finie du couple $\left(z_{1}, z_{2}\right)$.

Un exemple particulièrement important d'intégrale de Stieltjes est la fonction caractéristique :

$$
\varphi(s)=\int_{-\infty}^{+\infty} e^{i s z} d \mathrm{H}(z)
$$

La fonction caractéristique, toujours continue, caractérise la fonction de répartition de façon unique, comme le montre la formule de réciprocité :

$$
\mathrm{H}^{*}(b)-\mathrm{H}^{*}(a)=\lim _{s \rightarrow \infty} \frac{1}{2 \pi} \int_{-\mathrm{s}}^{+s} \frac{e^{-i s a}-e^{-i s b}}{i s} \varphi(s) d s
$$

où $H^{\star}$ est la fonction $H$ normalisée égale à : $\frac{H(z+0)+H(z-0)}{2}$ en tout point de discontinuité de $H$.

DE L'ADDITION DES VARIABLES ALÉatoIRES.

Soit une variable aléatoire $U$ de fonction de répartition $\mathrm{G}(u)$ et telle que :

$$
\mathrm{U}=\mathrm{Z}_{1}+\mathrm{Z}_{2}
$$

Il résulte du théorème des probabilités totales que l'on a :

$$
d \mathrm{G}(u)=\int_{z_{2}} d_{2} \mathrm{H}\left(u-z_{2}, z_{2}\right)
$$

Si, de plus, les variables $Z_{1}$ et $Z_{2}$ sont indépendantes en probabilité, on a :

$$
d \mathrm{G}(u)=\int_{z_{2}} d \mathrm{H}_{1}\left(u-z_{2}\right) d \mathrm{H}_{2}\left(z_{2}\right)
$$

Les fonctions caractéristiques vérifient alors la relation remarquable :

$$
\varphi_{u}(s)=\varphi_{z_{1}}(s) \cdot \varphi_{z_{1}}(s)
$$


SUR LES PROCESSUS STOCHASTIQUES.

Soit un processus aléatoire $\mathrm{X}(t)$ défini à des époques équidistantes. Si l'intervalle de temps constant est pris pour unité, on peut écrire :

$$
\ldots, \quad \mathrm{X}_{1}, \quad \mathrm{X}_{2}, \quad \ldots, \mathrm{X}_{t-1}, \quad \mathrm{X}_{t}, \mathrm{X}_{t+1}
$$

Ce processus est markovien si la loi de probabilité conditionnelle des états du processus aux époques $t+1$, $t+2 \ldots$, connaissant les états observés aux époques $t, t-1, t-2$, etc., ne dépend en fait que de l'état observé à l'époque $t$ et non pas des états observés aux époques antérieures.

Le traitement des processus non markoviens peut être abordé par la considération des processus markoviens imbriqués (imbedded Markov chains). Si de l'ensemble ordonné des instants $(. . ., t-1, t, t+1, \ldots)$ on peut extraire un sous-ensemble $(\ldots, \tau-1, \tau, \tau+1, \ldots)$ tel que le processus défini sur ce sous-ensemble est markovien, ce dernier permet l'étude du processus général.

Le procesisus est dit stationnaire si la loi de probabilité conjointe de l'ensemble des variables :

$$
\mathrm{X}_{t_{1}}, \quad \mathrm{X}_{t_{2}}, \quad \ldots, \quad \mathrm{X} t_{t_{1}}
$$

est identique à celle des variables relatives aux instants $t_{1}-\tau, t_{2}-\tau, \ldots, t_{k}-\tau$, quel que soit $\tau$.

- Le processus est dit ergodique si les moyennes temporelles de la forme :

$$
\frac{1}{\mathrm{~T}} \sum_{t=0}^{\mathrm{T}} \mathrm{X}_{t}
$$

ont un sens à la limite pour 'T grand. Si de nombreux processus rencontrés en pratique sont à la fois stationnaires et ergodiques et si, dans ce cas, la limite de la moyenne temporelle s'identifie à l'espérance mathématique de $\mathrm{X}_{t}$ pour $t$ quelconque, ces propriétés sont loin d'être la règle générale. Ces notions sont cependant essentielles. C'est l'ergodicité supposée des processus de débits ou de précipitations qui justifie l'usage courant, chez les hydrologues, de la prise en compte des moyennes temporelles pour caractériser les distributions de fréquences de ces grandeurs.

\section{Equations de base}

Revenons aux hypothèses de Moran que résument les équations (1). Il est souvent commode de raisonner non pas sur $Z$, mais sur la variable continue:

$$
\mathrm{U}_{t}=Z_{t}+\mathrm{X}_{t}
$$

Par ailleurs, si on définit une variable $V_{t}$ telle que :

$$
\begin{array}{lll}
\mathrm{V}_{t}=\mathrm{M} & \text { si } & \mathrm{U}_{t}<\mathrm{M} \\
\mathrm{V}_{t}=\mathrm{U}_{t} & \text { si } & \mathrm{M} \leqslant \mathrm{U}_{t}<\mathrm{K} \\
\mathrm{V}_{t}=\mathrm{K} & \text { si } & \mathrm{U}_{t} \geqslant \mathrm{~K}
\end{array}
$$

les équations (1) sont résumées en une seule :

$$
\mathrm{U}_{t+1}=\mathrm{V}_{t}+\mathrm{X}_{t+1}-\mathrm{M}
$$

Les fonctions de répartitions respectives $\mathrm{H}_{t}^{\prime}$ et $\mathrm{H}_{t}$ des couples $\left(\mathrm{X}_{t+1}, \mathrm{~V}_{t}\right)$ et $\left(\mathrm{X}_{t+1}\right.$, $\left.\mathrm{U}_{t}\right)$ sont alors liées par la relation :

avec :

$$
\mathrm{H}_{t}^{\prime}(x, u)=\mathrm{H}_{1} \int_{0}^{\mathrm{I}} d_{u} \mathrm{H}_{t}(x, u)+\mathrm{H}_{2} \int_{\mathrm{M}}^{\mathrm{K}} d_{u} \mathrm{H}_{t}(x, u)+\mathrm{H}_{3} \int_{\mathrm{K}}^{\infty} d_{u} \mathrm{H}_{t}(x, u)
$$

$$
\begin{aligned}
& \mathrm{H}_{1}=\left\{\begin{array}{lll}
0 & \text { si } \quad \mathrm{U}_{t}<\mathrm{M} \text { pour tout } x \\
1 & \text { si } \quad \mathrm{U}_{t} \geqslant \mathrm{M} \text { pour tout } x
\end{array}\right. \\
& \mathrm{H}_{2}= \begin{cases}0 & \text { si } \quad \mathrm{U}_{t}<\mathrm{M} \text { pour tout } x \\
& \frac{\mathrm{H}_{t}(x, u)-\mathrm{H}_{t}(x, \mathrm{M})}{\mathrm{H}_{t}(x, \mathrm{~K})-\mathrm{H}_{t}(x, \mathrm{M})} \text { si } \mathrm{M} \leqslant \mathrm{U}_{t}<\mathrm{K}\end{cases} \\
& \mathrm{H}_{3}=\left\{\begin{array}{lll}
\mathrm{si} \quad \mathrm{U}_{t} \geqslant \mathrm{~K} \text { pour tout } x \\
0 & \text { si } \quad \mathrm{U}_{t}<\mathrm{K} \text { pour tout } x
\end{array}\right.
\end{aligned}
$$

11 résulte immédiatement des formules (8) et (11) que la fonction de répartition $\mathrm{G}_{t+1}$ de $\mathrm{U}_{t+1}$ vérifie :

$$
d \mathrm{G}_{t+1}(x)=\int_{u} d_{2} \mathrm{H}_{t}^{\prime}(x+\mathrm{M}-u)
$$


soit, en utilisant (12) :

$$
d \mathrm{G}_{t+1}(x)=\int_{u=0}^{u=\mathrm{M}} d_{2} \mathrm{H}_{t}(x, u)+\int_{u=\mathrm{I}}^{u=\mathrm{K}} d_{2} \mathrm{H}_{t}(x+\mathrm{M}-u, u)+\int_{u=\mathrm{K}}^{u=\infty} d_{2} \mathrm{H}_{t}(x-\mathrm{K}+\mathrm{M}, u)
$$

On notera que toutes les variables étudiées sont positives ou nulles, de telle sorte que les fonctions de répartition définies ci-dessus sont nulles pour les valeurs négatives des arguments.

\section{Equation de Moran}

Dans le cas où les $\mathrm{X}_{t}$ successifs sont indépendants en probabilité, il en est de même des variables $\mathrm{U}_{t}$ et $\mathrm{X}_{t+1}$, de telle sorte que :

$$
d_{2} \mathrm{H}_{t}\left(x_{t+1}, u_{t}\right)=d \mathrm{~F}_{t+1}\left(x_{t+1}\right) d \mathrm{G}_{t}\left(u_{t}\right)
$$

où $\mathrm{F}_{t+1}$ est la fonction de répartition de $\mathrm{X}_{t+1}$.

Si le processus $X_{t}$ est stationnaire et si on se place assez loin du début de la gestion du réservoir de façon que l'influence des conditions initiales soit négligeable, le processus atteint un état stationnaire. Le fait qu'un tel état puisse être atteint peut être démontré rigoureusement; nous l'admettrons ici; on peut alors supposer que les lois de probabilité de chacune des variables $\mathrm{U}_{t+1}$ et $\mathrm{U}_{t}$ sont identiques. Dans ce cas, (13) permet d'écrire :

$$
d \mathrm{G}(x)=d \mathrm{~F}(x) \int_{0}^{\mathrm{M}} d \mathrm{G}(u)+\int_{u=\mathrm{I}}^{u=\mathrm{K}} d \mathrm{~F}(x+\mathrm{M}-u) d \mathrm{G}(u)+d \mathrm{~F}(x-\mathrm{K}+M) \int_{\mathrm{K}}^{\infty} d \mathrm{G}(u)
$$

Une intégration par parties permet l'obtention d'une équation intégrale du type de Volterra pour les fonctions de répartition $\mathrm{G}$ et $\mathrm{F}$ :

avec $f=d \mathrm{~F} / d x$.

$$
\mathrm{G}(x)=\mathrm{F}(x+\mathrm{M}-\mathrm{K})+\int_{\mathrm{X}}^{\mathrm{K}} \mathrm{G}(u) f(x+\mathrm{M}-u) d u
$$

La résolution de l'équation (15) permet notamment le calcul de :

- $G(M)$ : probabilité que le réservoir soit vide au début de la période $t$, ce qui, d'après la propriété ergodique, permet l'évaluation de la proportion du nombre de périodes où le réservoir est vide;

- 1-G(K) : probabilité de dépassement de la capacité du réservoir, e'est-à-dire la probabilité de dépassement du volume de sortie maximal admis.

L'équation (14) peut être résolue analytiquement dans le cas où la loi des apports F est une loi du type III de Pearson :

avec $\gamma$ entier positif.

$$
d \mathrm{~F}=\frac{\alpha^{\gamma}}{\Gamma(\gamma)} e^{-\alpha: x} x^{\gamma-1} d x
$$

La solution est cependant de forme assez complexe et caractérisée par la non analyticité de $d \mathrm{G} / d x$ (absence de dérivées pour certaines valeurs de $x$ ).

La fonction caractéristique $\varphi_{u}(s)$ peut être obtenue directement en utilisant la formule (14):

$$
\varphi_{u}(s)=\varphi_{u s}(s)\left[\int_{0}^{\mathrm{M}} d \mathrm{G}(u)+e^{-i s \mathrm{M}} \int_{\mathrm{M}}^{\mathrm{K}} e^{i s u} d \mathrm{G}(u)+e^{-i s(\mathrm{MI}-\mathrm{K})} \int_{\mathrm{KK}}^{\infty} d \mathrm{G}(u)\right]
$$

\section{La méthode de simulation}

Considérons le modèle simplifié de Moran et les hypothèses qui le conditionnent, notamment la stationnarité du processus $\mathrm{X}_{t}$. La prise en compte de la fonction de répartition $\mathrm{F}$ permet la construction d'une séquence de réalisations « fictives » du processus $\mathrm{X}_{t}$, soit :

$$
x_{1}, \quad x_{2}, \quad \ldots, \quad x_{i}, \quad x_{t+1}, \quad \ldots
$$

chaque $x_{i}$ est déterminé par $\mathrm{F}\left(x_{i}\right)=\mathrm{N}_{i} 10^{-k}$ où les $\mathrm{N}_{i}$ successifs sont des nombres « au hasard $»$ de $k$ chiffres tirés d'une table ou engendrés par un algorithme adéquat si on utilise une calculatrice électronique.

Sur la base de conditions initiales convenablement choisies, les équations (1) permettent alors la construction d'une séquence de réalisations « fictives » du processus $Z_{t}$, soit :

$$
z_{1}, \quad z_{2}, \quad \ldots, \quad z_{t}, \quad z_{i+1}, \quad \ldots
$$

L'absence d'influence des conditions initiales, hypothèse qui peut être admise à partir d'un seuil $t_{0}$ convenablement choisi, permet alors l'étude statistique de la distribution de $Z_{1}$ à partir de l'échantillon $: z_{t_{c}}, z_{t_{0}+1}$, $\ldots, z_{t_{0}+\mathrm{N}}$.

Le procédé est généralisable et peut s'appliquer très aisément aux cas de règles de gestion et de lois de 
probabilités des apports très complexes. L'hypothèse de stationnarité de ce processus n'est pas essentielle. On peut également traiter le cas des réservoirs multiples sans complications prohibitives.

On doit remarquer que les lois de probabilités des apports ne sont que des modèles mathématiques ajustés a priori aux observations réelles du processus $\mathrm{X}_{t}$. II est clair que ces lois de probabilité ne font que traduire, sans l'accrôttre, l'information contenue dans les observations. Dans ces conditions, on pourrait penser qu'il suffit d'effectuer la simulation sur les observations réelles.

Un tel raisonnement poussé à l'extrême amènerait à penser que la considération de modèles probabilistes des apports est sans objet. Ce serait, à notre sens, une erreur grave.

En effet, il ne faut pas oublier que, dans le cadre de la méthode, on n'atteint la distribution de $Z$ qu'à travers un échantillon de réalisations de cette variable. Il importe done de contrôler les erreurs d'échantillonnage pour connaitre la précision de nos estimations. Ce contrôle ne peut être fait que dans le cadre d'un modèle probabiliste adéquat, qui permet notamment la détermination du nombre de périodes nécessaires pour atteindre une précision donnée. Que la recherche d'une précision supérieure à celle que donnent les observations réelles soit illusoire, certes, mais encore importe-t-il de connaitre cette précision; c'est en fait le seul objet de la simulation à partir de réalisations fictives issues du modèle probabiliste ajusté et non pas à partir des observations réelles.

Utilisé de cette façon le procédé de simulation est très puissant. Il se peut cependant que le traitement de certains cas particuliers par des méthodes plus analytiques permette certaines économies en temps de calcul.

\section{dans le cas des apports liés en chaîne de Markoff stationnaire}

La non-stationnarité des processus des débits n'est pas un obstacle insurmontable à l'application pratique de la théorie de Moran au problème des crues. Par contre, cette théorie ne peut s'appliquer pour des périodes de temps d'assez courtes durées pour lesquelles la dépendance entre apports successifs est forte. Mais la théorie peut être aménagée pour tenir compte de cette dépendance.

Dans le cas de l'indépendance des apports $X_{t}$, les processus $U_{t}$ et $Z_{t}$ sont markoviens; il n'en est plus ainsi dans le cas où le processus $\mathrm{X}_{t}$ est lui-même markovien. Dans cette circonstance, la loi conditionnelle de $\mathrm{U}_{t+1}$, connaissant les états passés, ne dépend effectivement que des états $u_{t-1}$ et $u_{t}$ (chaîne double), de telle sorte que le processus imbriqué $u_{\tau}$ où $\tau$ repère les époques successives $t-2, t, t+2, t+4, \ldots$, devient markovien.

Ces considérations amènent à étudier non plus seulement les couples $\mathrm{X}_{t+1}, \mathrm{U}_{t}$, mais les triplets $\mathrm{X}_{t+1}, \mathrm{U}_{t}$, $\mathrm{X}_{t}$. On a alors :

Or :

$$
d_{2} \mathrm{H}_{t}\left(x_{t+1}, u_{t}\right)=\int_{r_{t}} d_{3} \mathrm{H}_{t}\left(x_{t+1}, u_{t}, x_{t}\right)
$$

$$
d_{3} \mathrm{H}_{t}\left(x_{t+1}, u_{t}, x_{t}\right)=d \mathrm{~F}_{t}^{*}\left(x_{t+1} / x_{t}\right) d \mathrm{G}_{t}{ }^{*}\left(u_{t} / x_{t}\right) d \mathrm{~F}_{t}\left(x_{t}\right)
$$

où l'astérisque indique les lois conditionnelles par rapport à $x_{t}$.

En effet, pour $x_{t}$ connu, les variables $\mathrm{X}_{t+1}$, et $\mathrm{U}_{t}$ sont indépendantes en probabilité, de sorte que la probabilité élémentaire conditionnelle du couple est égale au produit des probabilités élémentaires.

La clef $d u$ calcul repose sur le fait que la loi de $G_{t}{ }^{\star}{ }$ 'exprime directement en fonction de $d G_{t-1}$. En effet, d'après l'égalité (11) et sí $x_{t}$ est connu, $\mathbf{U}_{t}$ est fonction déterminée de $\mathbf{U}_{t-1}$, de sorte que le champ de variation de $\mathrm{U}_{t}$ est borné :

$$
x_{t} \leqslant \mathbf{U}_{t} \leqslant x_{t}+\mathrm{K}-\mathrm{M}
$$

les bornes sont prises avec les probabilités respectives:

$$
\int_{0}^{\mathrm{x}} d \mathrm{G}_{t-1} \quad \text { et } \quad \int_{\mathrm{K}}^{\infty} d \mathrm{G}_{t-1}
$$

et une valeur quelconque $u$ de l'intervalle est prise avec la probabilité élémentaire $d \mathrm{G}_{i-1}\left(u-x_{\mathrm{t}}+\mathrm{M}\right)$.

De façon plus formelle, on pose :

$$
\begin{aligned}
& -\mathrm{G}_{t-1}^{1}(u)=\left\{\begin{array}{ccc}
0 & \text { si } u<x_{t} \\
1 & \text { si } u \geqslant x_{t} &
\end{array} \text { - } \begin{array}{lll}
0 & \text { si } & u<x_{t}
\end{array}\right. \\
& -\mathrm{G}_{t-1}^{2}(u)=\left\{\begin{array}{lll}
\mathrm{G}_{t-1}\left(u-x_{t}+\mathrm{M}\right)-\mathrm{G}_{t-1}(\mathrm{M}) \\
\mathrm{G}_{t-1}(\mathrm{~K})-\mathrm{G}_{t-1}(\mathrm{M}) & \text { si } & x_{t} \leqslant u<x_{t}+\mathrm{K}-\mathrm{M} \\
1 & \text { si } & u \geqslant x_{t}+\mathrm{K}-\mathrm{M}
\end{array}\right. \\
& -\mathrm{G}^{3}{ }_{t-1}(u)=\left\{\begin{array}{lll}
0 & \text { si } & u<x_{t}+\mathrm{K}-\mathrm{M} \\
1 & \text { si } & u \geqslant x_{t}+\mathrm{K}-\mathrm{M}
\end{array}\right.
\end{aligned}
$$

Alors :

$$
d \mathrm{G}_{t}{ }^{\star}\left(u / x_{t}\right)=d \mathrm{G}_{t-1}^{x_{t-1}}(u) \int_{0}^{\mathrm{M}} d \mathrm{G}_{t-1}+d \mathrm{G}_{t-1}^{2}(u) \int_{\mathrm{M}}^{\mathrm{K}} d \mathrm{G}_{t-1}+d \mathrm{G}^{3}{ }_{t-1}(u) \int_{\mathrm{K}}^{\infty} d \mathrm{G}_{t-1}
$$




\section{J. BERNIER}

Le report de cette expression dans (17) et l'utilisation de (16) amènent au résultat suivant :

$$
\begin{aligned}
d_{2} \mathrm{H}_{t}(x, u)=d \mathrm{~F}_{i}^{*}(x / u) d \mathrm{~F}_{t}(u) \int_{0}^{\mathrm{M}} d \mathrm{G}_{t-1} & +\int_{v=u-\mathrm{K}+\mathrm{MI}}^{v=u} d \mathrm{~F}_{t}^{*}(x / v) d \mathrm{~F}_{t}(v) d \mathrm{G}_{t-1}(u-v+\mathbf{M}) \\
& \quad+d \mathrm{~F}_{t}^{*}(x / u-\mathbf{K}+\mathbf{M}) d \mathrm{~F}_{t}(u-\mathrm{K}+\mathbf{M}) \int_{\mathrm{K}}^{\infty} d \mathrm{G}_{t-1}
\end{aligned}
$$

L'ensemble de deux équations (13) et (20) permet le passage de $G_{t-1}$ à $G_{t+1}$.

La prise en compte des fonctions caractéristiques permet de préciser ces résultats dans le cadre d'un schéma de liaison stochastique particulier entre les $\mathrm{X}_{t}$ successifs.

Introduction des FOnCTIONS CARACTÉRistiques.

Posons:

$$
\begin{aligned}
\varphi_{t}(s) & =\int_{0}^{\infty} e^{i s \cdot t} d \mathrm{~F}_{t}(x) \\
\Gamma_{t}(s) & =\int_{0}^{\infty} e^{i s u} d \mathrm{G}_{t}(u) \\
\Psi_{t}(s) & =\int_{0}^{\infty} e^{i s y} d \mathrm{~F}_{t}^{*}(y / x)
\end{aligned}
$$

Nous supposons que la dépendance entre $\mathrm{X}_{t+1}$ et $\mathrm{X}_{t}$ est telle que :

$$
\mathrm{X}_{t+1}=\lambda x_{t}+\mathrm{W}_{t}
$$

où $W_{t}$ est une variable aléatoire, indépendante de $\mathrm{X}_{t}$, et dont la fonction caractéristique est $\Pi_{t}(s)$.

Ce schéma est un cas particulier de régression linéaire et il peut représenter avec suffisamment de réalisme la dépendance entre apports successifs à l'échelle journalière dans la majorité des cas.

Il résulte de (21) que :

$$
\Psi_{t}(s)=e^{i \lambda s x} \Pi_{t}(s)
$$

En multipliant (13) par $e^{i s x}$ et en intégrant sur $(0, \infty)$, on trouve en remplaçant $d_{2} \mathrm{H}(x, u)$ par sa valeur tirée de (20):

avec :

$$
\Gamma_{t+1}(s)=\Pi_{t}(s)\left[\varphi_{t}^{1}(s) \int_{0}^{\mathrm{M}} d \mathrm{G}_{t-1}+\varphi_{t}^{2}(s) \int_{\mathrm{M}}^{\mathrm{K}} d \mathrm{G}_{t-1}+\varphi_{t}^{3}(s) \int_{\mathrm{K}}^{\infty} d \mathrm{G}_{t-1}\right]
$$

$$
\begin{aligned}
\varphi_{t}{ }^{1}(s) & =\int_{0}^{\mathrm{M}} e^{\lambda i s u} d \mathrm{~F}_{t}(u)+e^{-i s \mathrm{M}} \int_{\mathrm{M}}^{\mathrm{K}} e^{i s u(1+\lambda)} d \mathrm{~F}_{t}(u)+e^{-i s(\mathrm{M}-\mathrm{K})} \int_{\mathrm{K}}^{\infty} e^{i s \lambda u} d \mathrm{~F}_{t}(u) \\
\varphi_{l}{ }^{2}(s) & =\frac{e^{-i s(1-\lambda) \mathrm{M}} \varphi_{t}^{1}(s) \int_{\mathrm{M}}^{\mathrm{K}} e^{(1-\lambda) i s v} d \mathrm{G}_{t-1}(v)}{\int_{\mathrm{M}}^{\mathrm{K}} d \mathrm{G}_{t-1}(v)} \\
\varphi_{t}{ }^{3}(s) & =e^{-i s(1-\lambda)(\mathrm{M}-\mathrm{K})} \varphi_{t}{ }^{1}(s)
\end{aligned}
$$

$\varphi^{i}(i=1,2,3)$ sont des fonctions caractéristiques, notamment $\varphi_{t}^{1}$ qui définit une variable aléatoire $y$ dont la probabilité élémentaire $d \mathrm{~L}_{t}(y)$ s'écrit immédiatement :

$$
\begin{array}{ll}
d \mathrm{~L}_{t}(y)=d \mathrm{~F}_{t}\left(\frac{y}{\lambda}\right) & \text { pour } 0 \leqslant y<\lambda \mathrm{M} \\
d \mathrm{~L}_{t}(y)=d \mathrm{~F}_{t}\left(\frac{y+\mathrm{M}}{1+\lambda}\right) & \text { pour } \lambda \mathrm{M} \leqslant y<(\lambda+1) \mathrm{K}-\mathrm{M} \\
d \mathrm{~L}_{t}(y)=d \mathrm{~F}_{t}\left(\frac{y+\mathrm{M}-\mathrm{K}}{\lambda}\right) & \text { pour } y \geqslant(\lambda+1) \mathrm{K}-\mathrm{M}
\end{array}
$$

Il en résulte que (22) s'écrit :

$$
\Gamma_{i+1}(s)=\mathrm{I}_{t}(s) \varphi_{t}{ }^{1}(s)\left[\int_{0}^{\mathrm{M}} d \mathrm{G}_{t-1}+e^{-i s(1-\lambda) \mathrm{M}} \int_{\mathrm{M}}^{\mathrm{K}} e^{i s(1-\lambda) v} d \mathrm{G}_{t-1}(v)+e^{-i s(1-\lambda)(\mathrm{M}-\mathrm{K})} \int_{\mathrm{K}}^{\infty} d \mathrm{G}_{t-1}\right]
$$

Le produit $\Pi_{t} \cdot \varphi_{t}{ }^{1}$ est la fonction caractéristique de la somme $\mathrm{W}_{t}+\mathrm{Y}$. Soit $d \mathrm{~F}_{t}^{\lambda}(x)$ la probabilité élémentaire de cette variable. L'inversion de la formule précédente donne: 


$$
\begin{aligned}
d \mathbf{G}_{t-1}(x)=d \mathrm{~F}_{t}^{\lambda}(x) \quad \int_{0}^{\mathrm{u}} d \mathrm{G}_{t-1}+ \\
\quad \int_{u=\mathrm{M}}^{u=\mathrm{K}} d \mathrm{~F}_{t}^{\lambda}[x+(1-\lambda)(\mathrm{M}-u)] d \mathrm{G}_{t-1}(u)+d \mathrm{~F}_{t}^{\lambda}\left[x+(1-\lambda)\left(\mathrm{M}-\mathrm{K}^{\lambda}\right] \int_{\mathrm{K}}^{\infty} d \mathrm{G}_{t-1}\right.
\end{aligned}
$$

Une intégration par partie donne le résultat plus condensé :

$$
\mathrm{G}_{t+1}(x)=\mathrm{F}_{t}^{\lambda}[x+(1-\lambda)(\mathrm{M}-\mathrm{K})]+(1-\lambda) \int_{u=\mathrm{X}}^{u=\mathrm{K}} d \mathrm{~F}_{t}^{\lambda}[x+(1-\lambda)(\mathbf{M}-u)] \mathrm{G}_{t-1}(u)
$$

Dans le cas des processus stationnaires, on obtient à la limite :

$$
\mathrm{F}_{t}=\mathrm{F}_{t+1} \quad \mathrm{G}_{t+1}=\mathrm{G}_{t}=\mathrm{G}
$$

de sorte que la fonction de répartition de $u$ est solution de l'équation intégrale :

$$
\mathrm{G}(x)=\mathrm{F}^{\lambda}[x+(1-\lambda)(\mathrm{M}-\mathrm{K})]+(1-\lambda) \int_{u=\mathrm{M}}^{u=\mathrm{K}} \mathrm{G}(u) d \mathrm{~F}^{\lambda}[x+(1-\lambda)(\mathrm{M}-u)]
$$

La comparaison des formules (15) et (25) montre que, dans ce cas particulier de schéma de dépendance, l'équation intégrale est de forme assez semblable à celle que l'on obtient dans le cas classique d'indépendance. La différence essentielle réside dans le fait que la loi des apports est remplacée par la loi d'une somme de variables indépendantes $\mathrm{W}+\mathrm{Y}$ :

- W dont la fonction caractéristique est $\Pi(s)$;

- Y dont la probabilité élémentaire est donnée par la formule (23).

\section{Etude des réservoirs à fins multiples}

La prise en compte de règles de gestion semblables à celles que résument les formules (2) où intervient une courbe d'alerte traduisant la gestion du réservoir en vue de la production d'énergie hydroélectrique ou de l'irrigation, ne soulève pas de difficultés importantes.

De préférence à la variable $Z$, il est encore commode d'utiliser la variable $U=Z+X$ et la probabilité $G_{t+1}$ de $\mathrm{U}_{t+1}$ est calculée à partir de la loi du couple $\mathrm{U}_{t}, \mathrm{X}_{t+1}$ par une formule du type (13):

$$
\begin{aligned}
d \mathrm{G}_{t+1}(x) & =\int_{u=0}^{u=k_{t}} d_{2} \mathrm{H}_{t}(x-u, u)+\int_{u=k_{t}}^{u=k_{t}+\mathrm{M}} d_{2} \mathrm{H}_{t}\left(x-k_{t}, u\right) \\
& +\int_{u=k_{t}+\mathrm{M}}^{u=\mathrm{K}} d_{2} \mathrm{H}_{t}(x+\mathrm{M}-u, u)+\int_{u=\mathrm{K}}^{u=\infty} d_{2} \mathrm{H}_{t}(x-\mathrm{K}+\mathrm{M}, u)
\end{aligned}
$$

A partir de cette formule, on peut appliquer mutatis mutandis la méthode du paragraphe VI qui amène au résultat suivant :

$$
\begin{aligned}
\mathrm{G}_{t+1}(x)=\mathrm{F}_{t+1}[x+(1-\lambda)(\mathrm{M}-\mathrm{K})]+(1-\lambda)\left\{\int_{u=0}^{u=k_{t}} \mathrm{G}_{t-1}(u) d \mathrm{~F}_{t-1}^{\lambda}[x-(1-\lambda) u]\right. & \\
& \left.+\int_{u=k_{t}+\mathrm{M}}^{\mathrm{K}} \mathrm{G}_{t-1}(u) d \mathbf{F}^{\lambda} t_{t-1}[x+(1-\lambda)(\mathbf{M}-u)]\right\}
\end{aligned}
$$

avec :

- $\mathrm{F}_{t^{\lambda}}$ fonction de répartition d'une somme de variables indépendantes $\mathrm{W}_{t}$ et $\mathrm{Y}_{t-1}^{t}$;

- $W_{t}$ de fonction caractéristique $\Pi_{t}(s)$ égale à celle de la variable $X_{t+1}$ liée par $X_{i}$;

- $\mathbf{Y}_{t-1}^{t}$ dont la probabilité élémentaire s'écrit :

$$
\begin{array}{lll}
d \mathrm{~L}_{t-1}^{t}(y)=d \mathrm{~F}_{t}\left(\frac{y}{1+\lambda}\right) & \text { si } & 0 \leqslant y<(1+\lambda) k_{t-1} \\
d \mathrm{~L}_{t-1}^{t}(y)=d \mathrm{~F}_{t}\left(\frac{y-k_{t-1}}{\lambda}\right) & \text { si } & (1+\lambda) k_{t-1} \leqslant y<(1+\lambda) k_{t-1}+\lambda \mathrm{M} \\
d \mathrm{~L}_{t-1}^{t}(y)=d \mathrm{~F}_{t}\left(\frac{y+\mathrm{M}}{1+\lambda}\right) & \text { si } & (1+\lambda) k_{t-1}+\lambda \mathrm{M} \leqslant y<(1+\lambda) \mathrm{K}-\mathrm{M} \\
d \mathrm{~L}_{t-1}^{t}(y)=d \mathrm{~F}_{t}\left(\frac{y+\mathrm{M}-\mathrm{K}}{\lambda}\right) & \text { si } & y \geqslant(1+\lambda) \mathrm{K}-\mathrm{M}
\end{array}
$$

La formule (27) définit une relation de récurrence entre $G_{t-1}$ et $G_{t+1}$ qui peuvent être ainsi calculées de 
proche en proche à partir de deux fonctions initiales $G_{1}$ et $G_{2}$ prises a priori et de la règle de gestion prise en compte, caractérisées par la séquence des seuils $k_{1}, k_{2}, \ldots, k_{t} \ldots$

Il est ainsi possible de comparer diverses règles de gestion par le calcul de leurs conséquences. Notamment dans le cas d'un réservoir mixte destiné conjointement à l'atténuation des crues au cours d'une période $\mathrm{T}$ et à l'irrigation après la période $\mathrm{T}$, la suite des $k_{t}$ définit la règle de remplissage au cours de la période et $k_{\mathrm{T}}$ le niveau visé à la fin de la période $T$ et correspondant au volume nécessaire à l'irrigation. On aura à tenir compte de :

$1^{\circ} \tau=\sum_{i=1}^{N}\left[1-G_{t}(K)\right]$ : espérance mathématique du nombre de périodes élémentaires au cours desquelles le volume de sortie est supérieur à la limite maximale $\mathrm{M}$ admise; $\tau / \mathrm{T}$ mesure alors la fréquence moyenne des cas de dépassement de cette limite;

$2^{\circ}$ La loi de probabilité $\mathrm{G}_{\mathrm{T}}(x)$ du niveau du réservoir au début de la période d'irrigation.

De façon semblable, la formule (27) peut servir à l'étude des conséquences de la gestion d'un réservoir destiné conjointement à l'atténuation des crues et à la production d'énergie électrique.

\section{Résolution par le système linéaire associé}

Pour les calculs numériques, il est commode de remplacer l'équation intégrale (27) par un système linéaire. Posons :

$$
\lambda=\frac{r}{s}(r \text { et } s \text { entiers })
$$

Dans la majorité des cas, on s'intéressera à des niveaux de la réserve égaux à de multiples entiers d'un certain volume unitaire V. Au prix d'un changement d'échelle on peut done supposer que $\mathrm{K}$, M et kt sont des entiers. Avec ces hypothèses on voit que les intégrales de (27) peuvent être approchées par des sommes si on prend des intervalles élémentaires d'amplitude égale à V/s et si les lois de probabilités des apports sont remplacées par des distributions de variables discrètes multiples entiers de cette unité $\mathrm{V} / s$.

Posons :

$$
\begin{aligned}
\mathrm{F}_{t}^{\lambda}\left(\frac{\mathrm{V}}{2 s}\right) & =\mathrm{P}^{t}[0], \quad \mathrm{F}_{t}^{\lambda}\left(\frac{3 \mathrm{~V}}{2 s}\right)-\mathrm{F}_{t^{\lambda}}\left(\frac{\mathrm{V}}{2 s}\right)=\mathrm{P}^{t}[1], \ldots \\
\mathrm{G}_{t}(x) & =\mathrm{R}^{t}[x]
\end{aligned}
$$

On aura, en notant que $[x]$ désigne le plus grand entier inférieur ou égal à $x$ :

$1^{\circ}$ si $j s \leqslant(s-r) k_{t}$ :

$$
\mathrm{R}^{t+1}[j s]=\frac{s-r}{s} \sum_{i=0,}^{[j s i(s-t)]} \mathrm{R}^{t-1}[i s] \mathrm{P}^{t-1}[j s-(s-r) i]
$$

$\underline{2^{\circ}}$ si $(s-z) k_{t}<j s \leqslant(s-r)(\mathrm{K}-\mathrm{M}):$

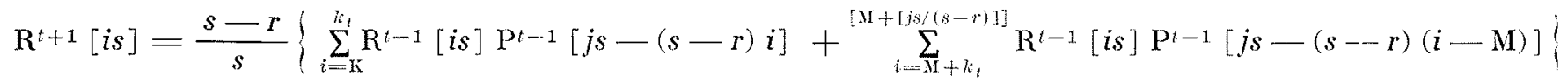

$\underline{3^{\circ} \text { si } j s>(s-r)(\mathrm{K}-\mathrm{M}):}$

$$
\begin{aligned}
\mathrm{R}^{t+1}[j s]= & \sum_{i=0}^{j s-(s-t)(\mathrm{K}-\mathrm{M})} \mathrm{P}^{i-1}[i]+ \\
& +\frac{s-r}{s}\left\{\sum_{i=0}^{k_{t}} \mathrm{R}^{t-1}[i s] \mathrm{P}^{t-1}[j s-(s-r) i]+\sum_{i=1 \mathrm{i}+k_{t}}^{i=\mathrm{K}} \mathrm{R}^{t-1}[i s] \mathrm{P}^{t-1}[j s-(s-r)(i-\mathrm{M})]\right\}
\end{aligned}
$$

Le système linéaire défini par les équations (29), (30), (31) fournit l'opérateur de passage du vecteur $\mathrm{R}^{t-1}$ au vecteur $\mathrm{R}^{t+1}$. L'équation (15) peut être ramenée à ce système en posant $k_{t}=0$ et en remarquant que, dans le cas stationnaire, on a $\mathrm{R}^{t+1}=\mathrm{R}^{t-1}=\mathrm{R}^{t}$, de telle sorte que la solution du problème est obtenue par la résolution d'un système linéaire classique.

Calcul des $\mathrm{P}^{t}[i]$.

L'inversion de $\Pi^{t}$ permet la détermination de la fonction de répartition de $\mathrm{W}_{t}$. Posons :

$$
\Pi_{0}{ }^{t}=\operatorname{Prob}\left[\mathrm{W}_{t}=0\right] \quad \Pi_{1}{ }^{t}=\operatorname{Prob}\left[0<\mathrm{W}_{t} \leqslant \frac{\mathrm{V}}{2 s}\right] \quad \Pi_{2}{ }^{t}=\operatorname{Prob}\left[\frac{\mathrm{V}}{2 s}<\mathrm{W}_{t} \leqslant \frac{3 \mathrm{~V}}{2 s}\right] \text { etc. }
$$


De façon semblable, les formules (28) permettent de poser:

$$
\begin{array}{rlrl}
l_{i}{ }^{t} & =\mathrm{F}_{t}\left[\frac{i \mathrm{~V}}{2(r+s)}\right]-\mathrm{F}_{t}\left[\frac{(i-1) \mathrm{V}}{2(r+s)}\right] & \text { si } & 0<i \leqslant(r+s) k_{t-1} \\
l_{i}{ }^{t}=\mathrm{F}_{t}\left[\frac{\left(i-\mathrm{k}_{t-1}\right) \mathrm{V}}{2 r}\right]-\mathrm{F}_{t}\left[\frac{\left(i-1-k_{t-1}\right) \mathrm{V}}{2 r}\right] & \text { si } & (r+s) k_{t-1}<i \leqslant(r+s) k_{t-1}+r \mathrm{M} \\
l_{i}{ }^{t}=\mathrm{F}_{t}\left[\frac{(i-\mathrm{M}) \mathrm{V}}{2(r+s)}\right]-\mathrm{F}_{t}\left[\frac{(i-1+\mathrm{M}) \mathrm{V}}{2(r+s)}\right] & \text { si } & (r+s) k_{t-1}+r \mathrm{M}<i \leqslant(r+s) \mathrm{K}-\mathrm{M} \\
l_{i}{ }^{t}=\mathrm{F}_{t}\left[\frac{(i+\mathrm{M}-\mathrm{K}) \mathrm{V}}{2 r}\right]-\mathrm{F}_{t}\left[\frac{(i-1+\mathrm{M}-\mathrm{K}) \mathrm{V}}{2 r}\right] & \text { si } & i>(r+s) \mathrm{K}-\mathrm{M}
\end{array}
$$

Avec ces conventions on a alors :

$$
\mathrm{P}^{t}[i]=\sum_{j=0}^{i-1} \Pi_{j}^{t} l_{i-j}^{i}
$$

L'ensemble des équations (29), (30), (31) et (32) permet la résolution concrète des problèmes pratiques.

\section{Sur un modèle stochastique des apports hydrauliques}

Dans une note récente (HYD-64/N' 43) nous avons décrit un modèle proposé par Halphen pour représenter le processus des débits. Rappelons-en les principales caractéristiques :

- La loi du débit $\mathrm{X}(t)$ à un instant $t$ est une loi du type III de Pearson :

$$
d \mathrm{~F}(x)=\frac{\alpha^{\gamma}}{\Gamma(\gamma)} e^{-\alpha x} x^{\gamma-1} d x
$$

- La variable $\mathrm{X}\left(t_{2}\right)$ liée par $\mathrm{X}\left(t_{1}\right)\left(t_{2}>t_{1}\right)$ est de la forme:

$$
\mathrm{X}\left(t_{2}\right)=\lambda x\left(t_{1}\right)+\mathrm{W}
$$

où $W$ est une variable aléatoire de fonction caractéristique :

$$
\Pi(s)=\left(\frac{\alpha-\lambda s}{\alpha-s}\right)^{\gamma}
$$

On a vu dans la note précitée que la loi $d Q(w)$ de W s'exprime comme une composition de lois de Pearson type III :

$$
d \mathbf{F}_{r}=\frac{[\alpha \lambda]^{r}}{\Gamma(r)} e^{-\alpha \lambda i v} w^{r-1} d w
$$

soit :

avec $P_{r}$ : probabilité binomiale négative :

$$
d \mathrm{Q}(w)=\sum_{r=0}^{\infty} \mathrm{P}_{r} d \mathrm{~F}_{r}
$$

$$
\mathrm{P}_{r}=\frac{\Gamma(\gamma+r)}{\Gamma(\gamma) \Gamma(r+1)} \lambda \gamma(1-\lambda)^{r}
$$

Dans le cadre de ce schéma, dont il est loisible de faire varier les paramètres en fonction du temps, le calcul des $p_{i}^{t}$ peut être entrepris.

Conclusions

Après un rappel des notions classiques de la théorie stochastique des réservoirs, nous avons présenté une méthode de résolution des problèmes où interviennent des apports dans les réservoirs, liés en chaîne de Marlkoff. La solution est valable dans le cas où la dépendance entre apports successifs vérifie un schéma linéaire simple. Le paragraphe IX a eu pour but de montrer qu'un tel schéma peut exister et qu'il peut représenter en première approximation les processus de débits.

L'algorithme de résolutions défini par les équations (29), (30), (31), (32) peut paraître compliqué; il est vrai qu'on ne peut espérer le traiter avec des moyens classiques que sur des cas très particuliers. Cependant un programme de calcul sur machine électronique est aisé à mettre en ouvre dans le cas présent; ce programme constitue la deuxième étape que nous avons en vue dans cette étude. 


\section{J. BERNIER}

Abstract

\section{Stochastic reservoir theory by J. Bernier *}

The purpose of stochastic-or conjectural-reservoir theory is to determine and study the probability law for the filling states of a reservoir-or a system of reservoirs - the inflows into which are the result of a given stochastic process, and the outflows from which are governed by a set operating policy which may be associated with water levels in the reservoir. Changes in reservoir state can be formally expressed by a system of equations (2) for a fairly general composite management rule.

Moran's well-known data are reviewed, which are based on the independence probability of the successive inflows, and on a very much simplified operating policy. Water level probability is then given by the integral equation of the form (15).

The simulation method available for more complex hypotheses is briefly described.

The main subject of the article is a description of a method for the calculation of reservoir water levels, based on the assumption of probable dependence of successive inflows, which are themselves assumed to be linked by a simple Markoff chain. In the case of the simplified operating rule, the probability law governing the reservoir water level and inflow for the same period can be calculated by the general formula (20). By allowing for a particular Markoff scheme described by formula (21), the water level relationship which is the solution of integral equation (25) can be calculated.

A similar method is applied to the case of a multi-purpose operating policy, which leads to formula (28).

Paragraph VIII of the article deals with the practical solution of the integral equations obtained. It is shown that these can be solved by replacing them by a linear system of fairly complex form (29), (30), (31) easily dealt with by a computer.

Paragraph IX describes a mode whereby the basic assumptions for the theory described in this article are verified, and which can give a fairly realistic representation of the stochastic processes associated with reservoir inflows.

\footnotetext{
* Ingénieur au Centre de Recherches et d'Essais de Chatou (E.D.F.).
} 